ACCEPTED MANUSCRIPT

\title{
Computational modeling of electrically conductive networks formed by graphene nanoplatelet-carbon nanotube hybrid particles
}

To cite this article before publication: Angel Mora et al 2018 Modelling Simul. Mater. Sci. Eng. in press https://doi.org/10.1088/1361-651X/aaab7a

\section{Manuscript version: Accepted Manuscript}

Accepted Manuscript is "the version of the article accepted for publication including all changes made as a result of the peer review process, and which may also include the addition to the article by IOP Publishing of a header, an article ID, a cover sheet and/or an 'Accepted

Manuscript' watermark, but excluding any other editing, typesetting or other changes made by IOP Publishing and/or its licensors"

This Accepted Manuscript is @ 2018 IOP Publishing Ltd.

During the embargo period (the 12 month period from the publication of the Version of Record of this article), the Accepted Manuscript is fully protected by copyright and cannot be reused or reposted elsewhere.

As the Version of Record of this article is going to be / has been published on a subscription basis, this Accepted Manuscript is available for reuse under a CC BY-NC-ND 3.0 licence after the 12 month embargo period.

After the embargo period, everyone is permitted to use copy and redistribute this article for non-commercial purposes only, provided that they adhere to all the terms of the licence https://creativecommons.org/licences/by-nc-nd/3.0

Although reasonable endeavours have been taken to obtain all necessary permissions from third parties to include their copyrighted content within this article, their full citation and copyright line may not be present in this Accepted Manuscript version. Before using any content from this article, please refer to the Version of Record on IOPscience once published for full citation and copyright details, as permissions will likely be required. All third party content is fully copyright protected, unless specifically stated otherwise in the figure caption in the Version of Record.

View the article online for updates and enhancements. 


\title{
REGULAR ARTICLE
}

\section{Computational modeling of electrically conductive networks formed}

\section{by graphene nanoplatelet-carbon nanotube hybrid particles}

A. Mora ${ }^{\mathrm{a}}$, F. $\operatorname{Han}^{\mathrm{a}}$, and G. Lubineau ${ }^{\mathrm{a}}$

${ }^{a}$ COHMAS Laboratory, King Abdullah University of Science and Technology (KAUST),

Thuwal, Saudi Arabia

\section{ARTICLE HISTORY}

Compiled January 29, 2018

\begin{abstract}
One strategy to ensure that nanofiller networks in a polymer composite percolate at low volume fractions is to promote segregation. In a segregated structure, the concentration of nanofillers is kept low in some regions of the sample. In turn, the concentration in remaining regions is much higher than the average concentration of the sample. This selective placement of the nanofillers ensures percolation at low average concentration. One original strategy to promote segregation is by tuning the shape of the nanofillers. We use a computational approach to study the conductive networks formed by hybrid particles obtained by growing carbon nanotubes (CNTs) on graphene nanoplatelets (GNPs). The objective of this study is (1) to show that the higher electrical conductivity of these composites is due to the hybrid particles forming a segregated structure and (2) to understand which parameters defining the hybrid particles determine the efficiency of the segregation. We construct a microstructure to observe the conducting paths and determine whether a segregated structure has indeed been formed inside the composite. A measure of efficiency is presented based on the fraction of nanofillers that contribute to the conductive network. Then, the efficiency of the hybrid-particle networks is compared to those of three other networks of carbon-based nanofillers in which no hybrid particles are used: only CNTs, only GNPs, and a mix of CNTs and GNPs. Finally, some parameters of the hybrid particle are studied: the CNT density on the GNPs, and the CNT and GNP geometries. We also present recommendations for the further
\end{abstract}

Corresponding author G. Lubineau. Email: gilles.lubineau@kaust.edu.sa 
improvement of a composite's conductivity based on these parameters.

\section{KEYWORDS}

Graphene nanoplatelet; Carbon nanotube; Polymer composites; Segregated structure; Hybrid particle; Electrical properties

\section{Introduction}

Carbon nanotubes (CNTs) and graphene nanoplatelets (GNPs) possess high electrical conductivities, making them suitable nanofillers for improving the electrical conductivity of polymeric materials [1-4]. Polymer nanocomposites loaded with carbon-based conductive nanofillers are a promising technology for use in transparent electrodes [57]. Polymer composites whose piezoresistive behavior has been enhanced with carbonbased conductive fillers can be used for strain sensing or structural health monitoring applications [8-11].

The addition of conductive nanofillers to a polymer mattrix improves its electrical behavior through a percolation process, where nanofillers form conductive networks [12]. The filler concentration at which the electrical conductivity increases sharply by orders of magnitude is the percolation threshold, which indicates that conductive networks have formed. However, the nanofillers need to be in close proximity to each other to form these conductive networks. There is a maximum distance, in the order of a few nanometers, above which conduction between nanofillers is no longer possible $[13,14]$. Due to this limitation, nanofillers that are too far apart will not join the conductive network.

Segregated structures that confine the nanofillers to specific locations in a composite have been reported $[15,16]$. This greatly reduces the percolation threshold and increases electrical conductivity compared to random structures, by improving the likelihood that the nanofillers will be in contact with each other $[17,18]$. For example, nanofillers in conductive polymers can be placed at the boundaries of the polymer granules $[17,19$, 20]. Although this improves the electrical properties of the polymer, boundary defects are introduced, which degrade the mechanical properties [17] and limits the use of segregated structures in sensing or structural health monitoring applications. 
Recently, a hybrid particle was developed to improve the electrical properties of polymers $[21,22]$. This hybrid particle is obtained by growing CNTs directly on the surfaces of GNPs. Polymers doped with this hybrid particle had a higher electrical conductivity than those doped with either CNTs or GNPs alone, or with a mixture of these fillers [21,23]. Piezoresistive behavior was also demonstrated in polymers doped with these hybrid particles, which makes them a promising nanofiller for strain-sensing applications [24]. To explain the high electrical conductivity of polymers doped with hybrid particles, Zhao and Bai [24] proposed that the GNPs act as wire concentrators. Understood this way, each hybrid particle is a bundle of connected CNTs. Then, only one CNT from each bundle needs to be connected to the network for all of the CNTs to be connected, resulting in the formation of a conductive network at a lower nanofiller loading. In other words, the hybrid particles form a segregated structure in which the GNPs constrain the location of CNTs. Unfortunately, it is difficult to verify in an experimental setting that the hybrids have formed a segregated structure.

Therefore, we introduce a computational approach to study hybrid-particle networks and confirm that they do form segregated structures. This is the first time a computational approach has been applied to study these hybrid particles. In section 2, we describe the model used to generate the computational representations of the hybrid particles and present the procedure for turning the network of hybrid particles into a network of resistors. We use this resistor network to compute the conductivity of the composite. In section 3 , we present our results, where we prove that the hybrid-particle networks are segregated structures. In previous work, we developed a computational tool that allowed us to quantify the efficiency of a conductive network formed by conductive nanofillers [25]. Using this tool, we show that the hybrid-particle networks are more efficient and result in more conductive networks than three other types of nanofillers: CNTs, GNPs, and a mix of CNTs and GNPs. In addition, we study some parameters of the hybrid particle in order to further improve the composite's conductivity: the CNT density on GNPs, and the CNT and GNP geometries. Finally, we draw conclusions in section 4. 


\section{Numerical modeling}

\subsection{Geometric modeling of a hybrid-particle network}

Each hybrid particle consists of a GNP and several CNTs, which are generated computationally. The GNP is approximated as a thin parallelepiped with a square base. In this way, the geometry of the GNP is defined by its side length, $l_{G N P}$, and thickness, $t_{G N P}$, as shown in Figure 1a. Each generated CNT is comprised of $n+1$ points (labeled $s_{0}$ to $s_{n}$ in Figure $1 \mathrm{~b}$ ) defining $n$ segments with length $l$ and radius $r_{C N T}$ [26]. The point $s_{0}$ is randomly generated on the surface of the GNP. To generate the point $s_{i+1}(i=0,1,2, \ldots, n-1)$, a local Cartesian coordinate system $\left(\boldsymbol{x}_{i}, \boldsymbol{y}_{i}, \boldsymbol{z}_{i}\right)$ with origin at $s_{i}$ is defined. When $i=0$, the local coordinate system, $\left(\boldsymbol{x}_{i}, \boldsymbol{y}_{i}, \boldsymbol{z}_{i}\right)$, is the same as the global coordinate system, $(\boldsymbol{x}, \boldsymbol{y}, \boldsymbol{z})$ (see Figure $1 \mathrm{~b}$ ). For $i \geq 1, \boldsymbol{z}_{i}$ has the same direction as $\overrightarrow{s_{i-1} s_{i}}$ and $\boldsymbol{x}_{i}$ is the projection of $\boldsymbol{x}$ on $A_{i}$, where $A_{i}$ is the plane containing the point $s_{i}$ with normal vector $\boldsymbol{z}_{i}$ (see Figure $1 \mathrm{~b}$ ). Then, the point $s_{i+1}$ has spherical coordinates $\left(l, \omega_{s}, \psi_{s}\right)$ in the local coordinate system $\left(\boldsymbol{x}_{i}, \boldsymbol{y}_{i}, \boldsymbol{z}_{i}\right)$. By choosing different specifications for the ranges and probability functions for the angles $\left(\omega_{s}, \psi_{s}\right)$, CNTs with different curliness can be generated $[25,26]$.

The number of CNTs per GNP surface, $n_{C N T}$, is calculated from the CNT/GNP mass ratio in the composite, $M$. The mass ratio is used as a parameter to control the amount of CNTs relative to GNPs in experiments. Assuming that every hybrid particle also has mass ratio $M$, we have that:

$$
M=\frac{2 n_{C N T} D_{C N T} V_{C N T}}{D_{G N P} V_{G N P}}
$$

where $D_{G N P}=2.0 \mathrm{~g} / \mathrm{cm}^{3}[27,28]$ and $D_{C N T}=1.8 \mathrm{~g} / \mathrm{cm}^{3}[29,30]$ are the densities for the GNPs and CNTs, respectively; and $V_{G N P}$ and $V_{C N T}$ are the volumes of a single GNP and a single CNT, respectively. The factor of 2 is included because CNTs are grown on both surfaces of the GNP.

A comparison between a single hybrid particle obtained experimentally [23] and one generated computationally is presented in Figure 2. The lengths of the CNTs typically 
a)
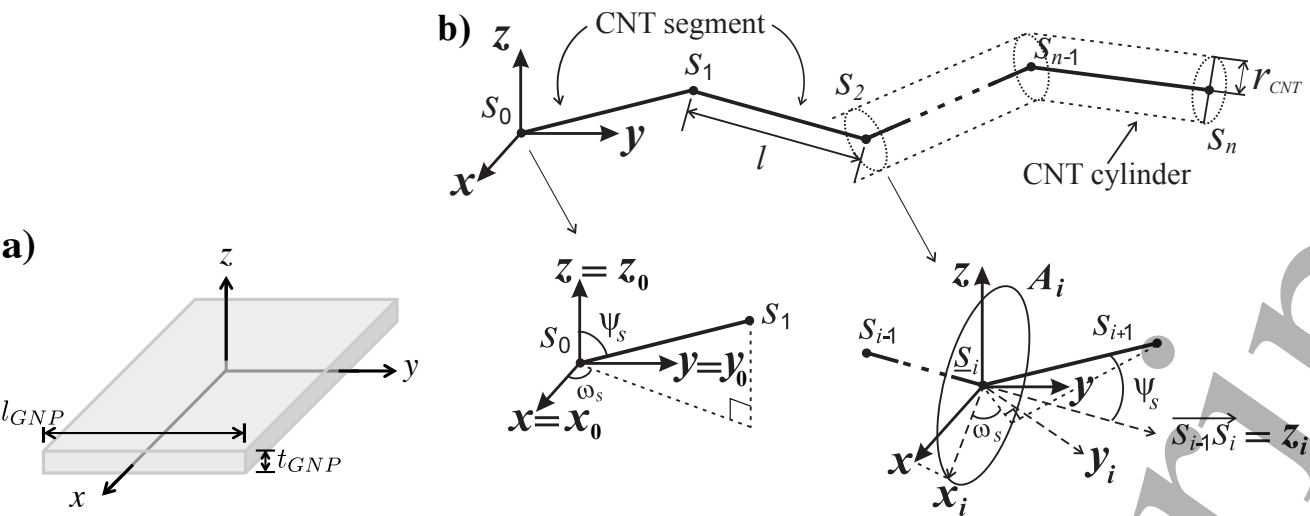

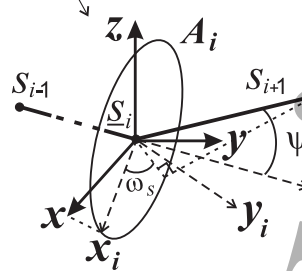

Figure 1. Geometric parameters of (a) a GNP and (b) a CNT.

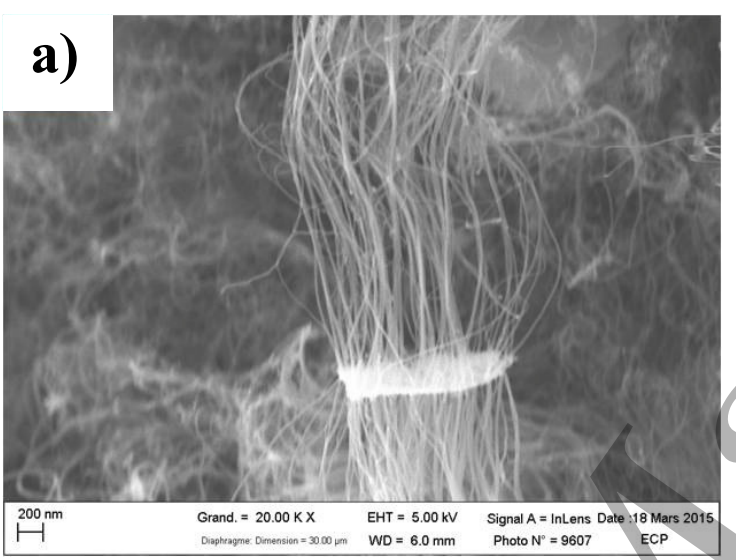

b)

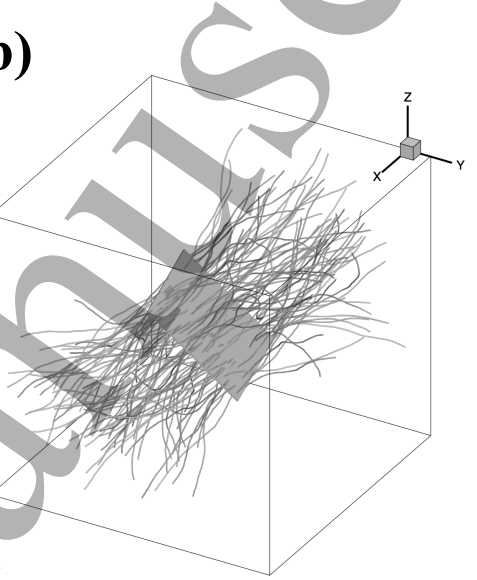

Figure 2. Examples of (a) an experimentally observed particle [23], and (b) a particle generated in a cubic sample with side length of $5 \mu \mathrm{m}$ with $l_{C N T}=3 \mu \mathrm{m}, r_{C N T}=10 \mathrm{~nm}, l_{G N P}=2 \mu \mathrm{m}$ and $t_{G N P}=30 \mathrm{~nm}, l=$ $10 \mathrm{~nm}$, and $\psi_{s}$ follows a normal distribution in the range $[-\pi / 12, \pi / 12]$

range between 2 and $4 \mu \mathrm{m}$, and their radii range from 7.5 to $10 \mathrm{~nm}[24,31]$. The GNPs have a side length of 1 to $4 \mu \mathrm{m}$ and thickness of around $30 \mathrm{~nm}$ [24,31]. Figure $2 \mathrm{~b}$ shows a particle generated in a cubic sample with side length of $5 \mu \mathrm{m}$ with $l_{G N P}=2$ $\mu \mathrm{m}, t_{G N P}=30 \mathrm{~mm}, l_{C N T}=3 \mu \mathrm{m}, r_{C N T}=10 \mathrm{~nm}, l=10 \mathrm{~nm}$, and $\psi_{s}$ follows a normal distribution in the range $[-\pi / 12, \pi / 12]$. The particles generated from our geometric model imitate the ones synthesized in experiments very well. From Figure 2a, we also observe that the concentration of CNTs on the GNP causes them to curl less than randomly dispersed CNTs. This can also be observed from images presented in references [23] and [24].

We generate the network of hybrid particles inside a cubic sample of size $[a \times a \times a]$. 

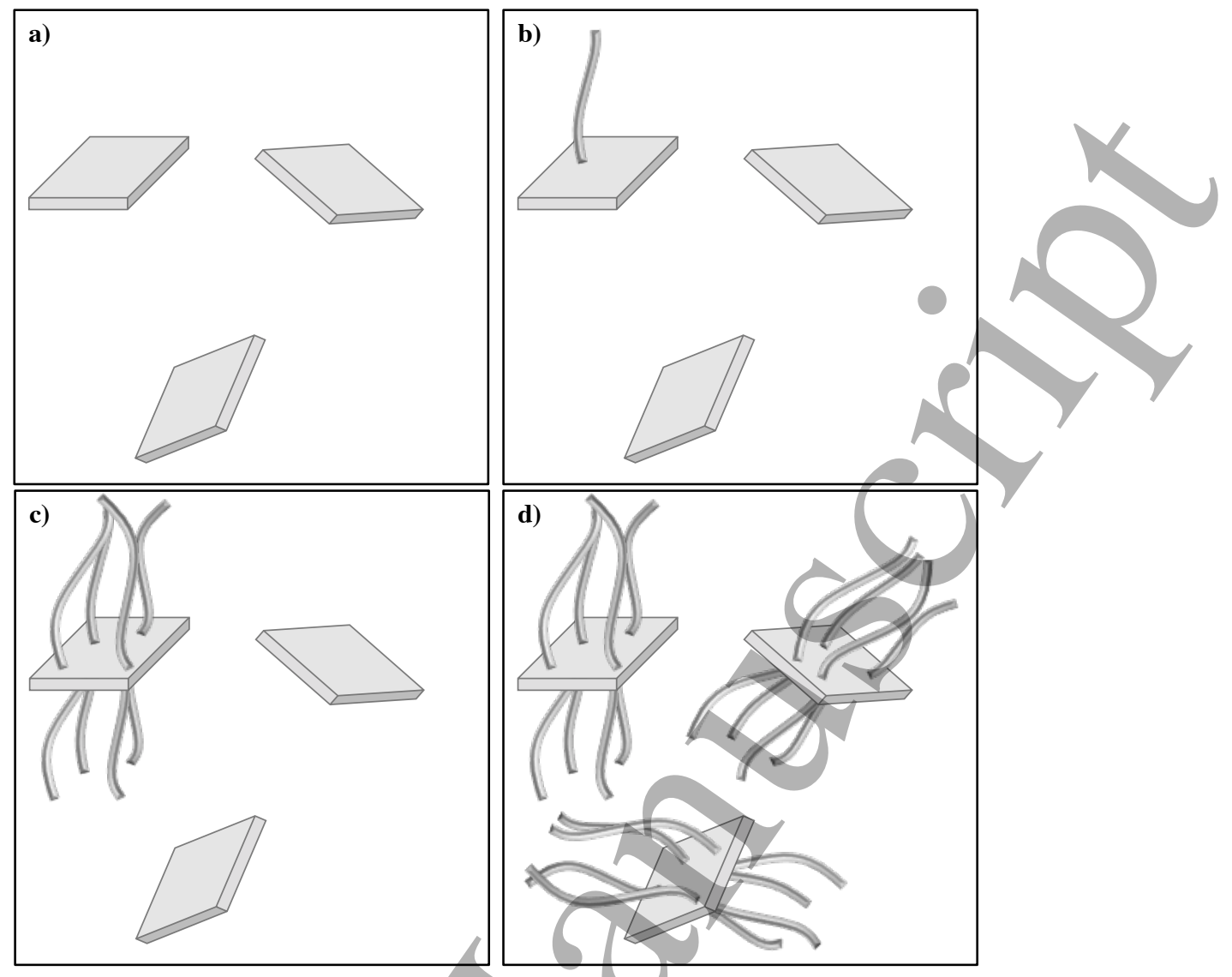

Figure 3. Schematic representation of the hybrid-particle network generation. The parallelepipeds represent GNPs and the cylinders, CNTs. (a) Generation of GNPs; (b) generation of a single CNT on a GNP; (c) all of the CNTs on one GNP are generated; and (d) a finished network of hybrid particles.

The process of generating a network of hybrid particles is schematically represented in Figure 3. First, using the mass ratio and the desired carbon content, the volume of GNPs and the number of GNPs needed to reach this volume are calculated. The resulting number of GNPs are generated and assigned to random locations and orientations inside the cubic sample (see Figure 3a). After the GNPs are generated, the CNTs are grown one by one on the GNP surfaces. All of the CNTs corresponding to a GNP are grown before moving on to the next GNP (see Figures 3b and 3c). Each time a full hybrid particle is generated (i.e., one GNP and all its CNTs), the volume fraction of generated particles in the sample is calculated and compared against the desired carbon content. We note that, when generating CNTs and GNPs, part of their volume might fall outside the cubic sample. In such cases, only the volume inside the cubic sample is considered. In particular, the CNT segments lying outside the cube 
are removed and the point lying at that boundary is calculated from the segment that intersects a boundary. In the case of GNPs, a plane at half the thickness (and parallel to the surfaces for CNT growth) is discretized as a set of points. Then, the volume of GNPs inside the cube is approximated as the fraction of points inside the cube. Once the required carbon content is reached, the generation process of the hybrid-particle network is finished (see Figure 3d). We allow the interpenetration of the hybrid particles because (1) the computations when generating the hybrid-particle networks are reduced and (2) our previous work showed that allowing interpenetrations of CNTs has a negligible effect on the fraction of nanofillers that form the conductive network $[25]$.

\subsection{Efficiency of the hybrid-particle network}

In our previous work, we developed a computational tool that allowed us to quantify the fraction of nanofillers that form the backbone of the network [25]. The backbone is defined as the current-carrying path of a conductive network of nanofillers. Here, we summarize the procedure for finding the backbone and how we use it to define efficiency.

First, the Hoshen-Kopelman algorithm [32,33] is used to find and group all of the particles having electrical contact into clusters. We consider two particles to be in electrical contact when they are separated by a distance less than a given threshold, $d_{t}=1.8 \mathrm{~nm}$ [13]. This $d_{t}$ is the maximum distance below which electron transfers occur due to quantum mechanical tunneling or electron hopping [34].

Then, the percolated clusters are found, i.e., the clusters that connect two opposite boundaries of a cubic sample. From each percolated cluster, we extract the backbone. This is done by applying the direct electrifying algorithm (DEA) [35,36], which uses the current-carrying definition of the backbone to determine zero-current pathways. In this algorithm, the network of hybrid particles is turned into a resistor network. Three types of resistors are considered in this network: junction resistors, CNT resistors, and GNP resistors.

A junction resistor is added when two hybrid particles are in electrical contact. Thus, a junction resistor might be found between two CNTs, two GNPs, or one CNT 


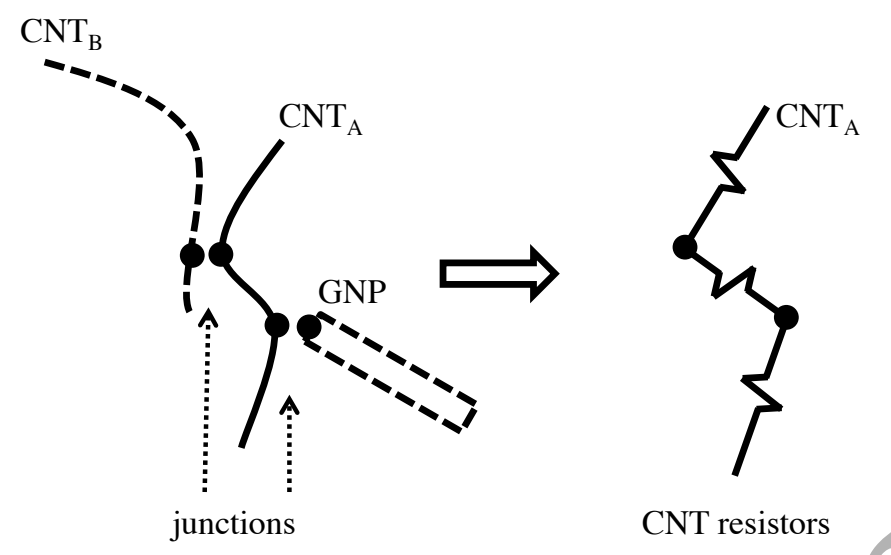

Figure 4. Example of a CNT with two junctions: one junction with $\mathrm{CNT}_{\mathrm{B}}$ and one junction with a GNP. $\mathrm{CNT}_{\mathrm{A}}$ results in three CNT resistors.

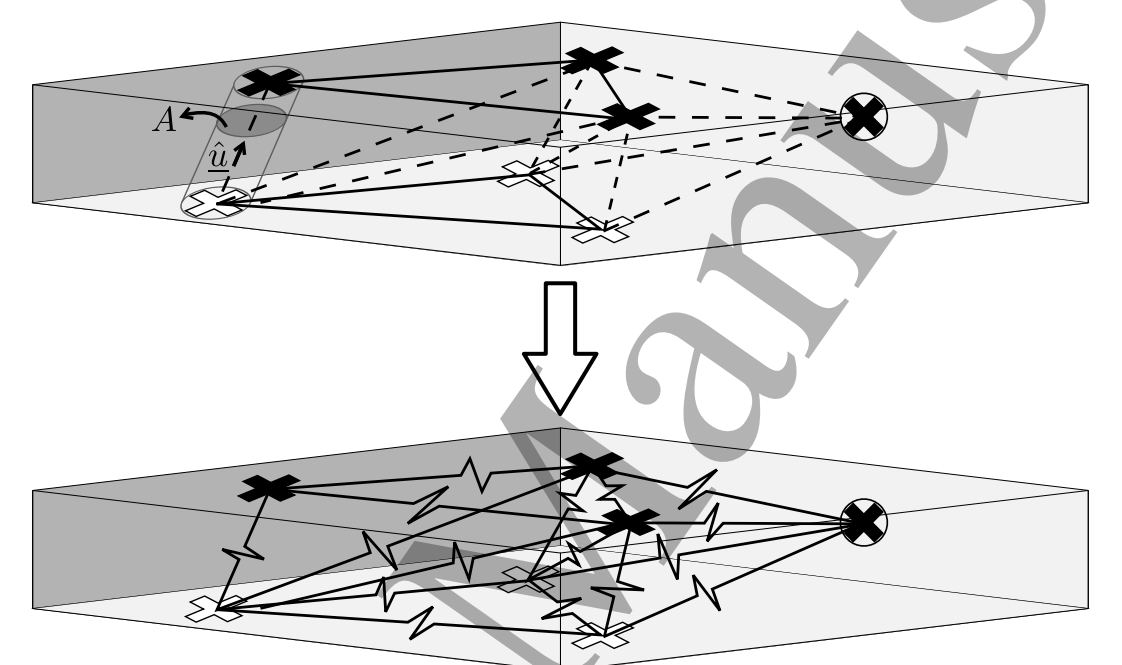

CNT growth point on top surface

$\approx$ CNT growth point on bottom surface

Junction point on lateral surface

- Edges on surfaces

- Edges through the thickness

Figure 5. Example of a triangulation formed by three CNT growth points on each GNP surface, top and bottom, and a junction point on a lateral surface. Each triangulation edge becomes a resistor. In the top GNP, $\underline{\hat{u}}$ is the unit vector along the triangulation edge and $A$ is the area of the conduction channel represented by a cylinder.

and one GNP.

A CNT resistor comes from the intrinsic resistance of a CNT. The number of CNT resistors will depend on the number of junctions on a given CNT. A CNT with $n$ junctions will result in $n+1 \mathrm{CNT}$ resistors in series. An example is given in Figure 4, 
where a CNT with two junction points results in three CNT resistors in series.

Each GNP forms a network of resistors, since conduction happens along the surface and through the thickness of the GNP. The CNT growth points on the GNP surfaces form a Delaunay triangulation $[37,38]$. If there are junction points with other CNTs or GNPs, then these points are also included in the triangulation. Each triangulation edge is considered to be a GNP resistor. An example of a triangulation using six CNT growth points and a junction is shown in Figure 5.

To find the backbone, voltage is applied and the electrical equations that define the voltage distribution over the resistor network are solved. The current passing through each resistor is calculated and those resistors with a non-zero current are part of the backbone. Here we note that the electrically conductive backbone is defined as the current-carrying member of the percolated cluster $[12,35,36]$. Thus, the definition of the backbone is physical in foundation. On the other hand, the problem of extracting the backbone from a resistor network is purely a mathematical problem that requires finding the non-conducting branches. Thus, for the purpose of extracting the backbone, all resistors are set to $1 \Omega$. This reduces the number of iterations required to solve the system of equations compared to using the actual values of the different types of resistors. For example, a CNT resistor is around $1.2 \times 10^{5} \Omega$ [25], while a junction resistor reaches up to $10^{18} \Omega[13,39]$. Having values with such differences in magnitude in the same matrix causes it to be ill conditioned. Thus, by using the same value for all resistors, the conditioning number of the matrix is improved. The results are not affected since the backbone is defined by the resistors with a non-zero current, not the magnitude of that current. We also note that some of the branches will bear high current and some low current due to the real local values of the junctions. However, this does not change the structure of the network itself. In our future work, we will include a non-linear definition of the junction resistance to account for this.

Finally, since only the backbone actually carries electricity in a hybrid-particle network, we define efficiency, $f_{e f f}$, as the fraction of hybrid particles that belong to the backbone: 


$$
f_{\text {eff }}=\frac{V_{\text {backbone }}}{V_{\text {tot }}}
$$

where $V_{\text {backbone }}$ is the volume of hybrid particles that belong to the backbone and $V_{\text {tot }}$ is the total volume of hybrid particles inside the cubic sample.

\subsection{Electrical conductivity of composites filled with hybrid particles}

Below the percolation threshold, the electrical conductivity of the polymer matrix plays an important role in the composite's conductivity. In this case, a micromechanics model (e.g. $[40,41])$ is more suitable for calculating the electrical conductivity of the composite than a model based on the conductivity of the nanofiller network. Above the percolation threshold, electrical conductivity is due mainly to the nanofiller network because of the large differences between the nanofiller and matrix conductivities. Therefore, we assume the electrical conductivity of the nanocomposite to be that of the nanofiller network.

The network conductivity is obtained by first cálculating its electrical resistance. The DEA is applied to the backbone to determine the equivalent electrical resistance of the resistor network, $R_{e q}$. For the purpose of calculating $R_{e q}$, we discard the 1 $\Omega$ resistors used to find the backbone and instead calculate the magnitudes of the junction resistors, CNT resistors, and GNP resistors as follows.

The magnitude of the junction resistance has been approximated in the literature as an exponential function that depends on the separation between nanofillers [13,42]. However, other factors influencing the magnitude of the junction resistance include the nanotube type, nanotube diameter, junction area, and polymer type [43-45]. This makes it difficult in practice to accurately determine the magnitude of the junction resistance. To overcome this difficulty, the use of a constant value or values obtained from a probability distribution function was proposed [13,43,46-48]. Given that some junction resistances have a magnitude close to those of CNT resistors [25], it is difficult to capture the effect of junction resistance in our model. For this reason, we use a constant value for the junction resistance that is higher than that of the CNT resistors 
in the network.

The resistance of a CNT resistor is calculated based on its geometry and the reported values of CNT conductivity. In the case of GNP resistors, the triangulation edges in a GNP might result in a resistor connecting two points through the thickness of the GNP. For example, a triangulation edge connects the two leftmost CNT growth points on the bottom and top surfaces of the GNP in Figure 5. Because of this, the tensorial representation of the GNP resistivity must be considered. The electrical/resistance of a triangulation edge is calculated as

$$
R_{G N P}=\frac{l_{\text {edge }}}{A} \underline{\hat{u}} \cdot \underline{\underline{\rho}} \cdot \underline{\hat{u}}
$$

where the $\underline{\rho}$ is the GNP resistivity tensor, $l_{\text {edge }}$ is the triangulation edge length, $\underline{\hat{u}}$ is the unit vector along the triangulation edge, and $A$ is the cross-sectional area of the conduction channel. The conduction channel is represented by the cylinder in Figure 5, where $\underline{\hat{u}}$ and $A$ are also indicated. Here, the resistivity tensor has the form

where $\rho_{s}$ and $\rho_{t}$ are the surface and through-thickness resistivities, respectively.

Next, the total current throughout the network is calculated and, using Ohm's law, the equivalent resistance, $R_{e q}$, of the network is obtained. Finally, the electrical conductivity of the sample, $\sigma$, is obtained as

where $l_{\text {sample }}=a$ is the side length of the cubic sample and $A_{\text {sample }}=a^{2}$ its cross-

$$
\sigma=\frac{l_{\text {sample }}}{R_{\text {eq }} A_{\text {sample }}}=\frac{1}{a R_{e q}}
$$


a)

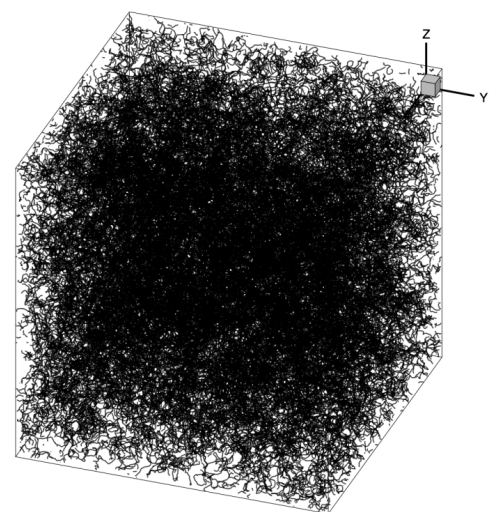

15

16 In all cases, the nanofiller content is $0.25 \%$ volume fraction.

sectional area.

\section{Results and discussion}

b)

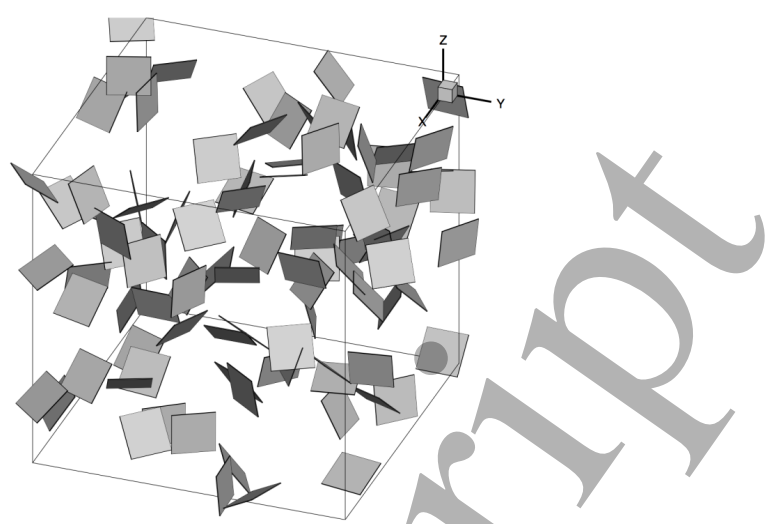

d)

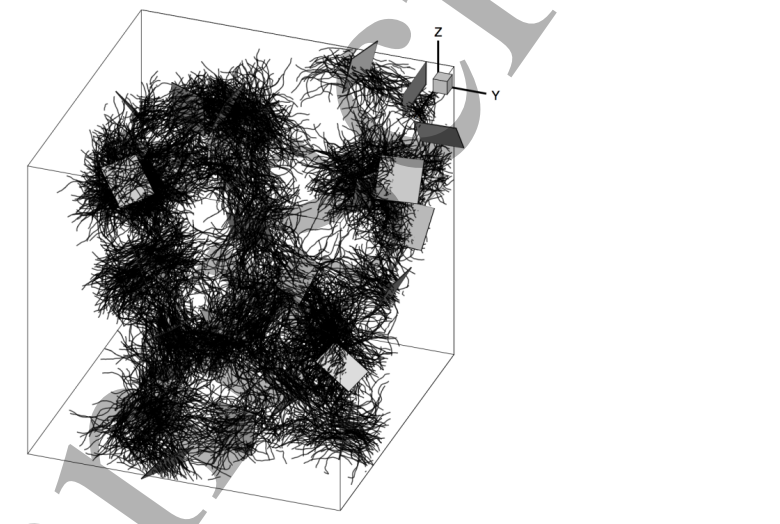

Figure 6. Networks formed using (a) CNTs, (b) GNPs, (c) mixed CNTs and GNPs, and (d) hybrid particles.

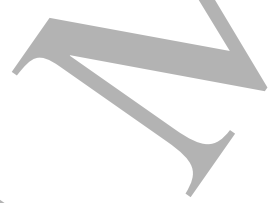

\subsection{Hybrid-particle network as a segregated structure}

In Figure 6, we present networks of CNTs, GNPs, mixed CNTs and GNPs, and hybrid particles. The CNTs and GNPs were generated with the same geometry as that presented in Figure 2b. In all cases, the cubic samples are of size $a=15 \mu \mathrm{m}$ and the nanofiller content is $0.25 \%$ volume fraction. The samples of pure CNTs, GNPs, and a mix of CNTs and GNPs show homogeneous distributions of the nanofillers (Figures $6 \mathrm{a}, 6 \mathrm{~b}$, and $6 \mathrm{c}$, respectively). However, the distribution of the hybrid particles (Figure $6 \mathrm{~d}$ ) is not homogeneous, since the CNTs are grown in concentrated 'bundles' on the GNPs. Thus, the regions close to the GNP surfaces have a higher CNT content. The hybrid-particle network consists of paths formed by these CNT bundles. 
a)

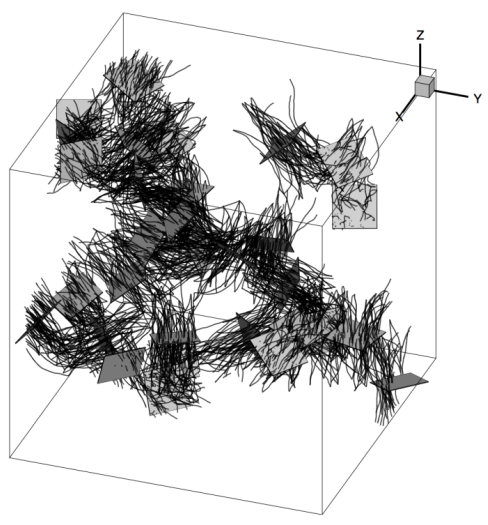

b)

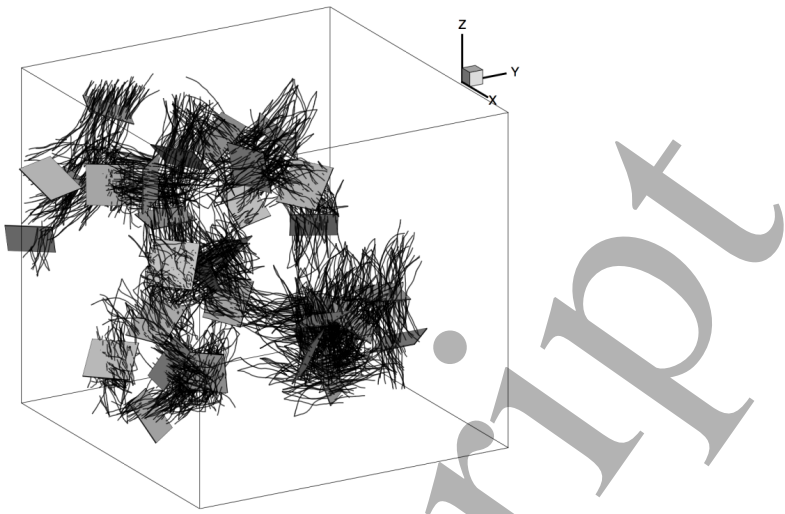

Figure 7. (a) Backbone of a hybrid-particle network from the sample shown in Figure 6d. (b) Rotated view of (a).

a)
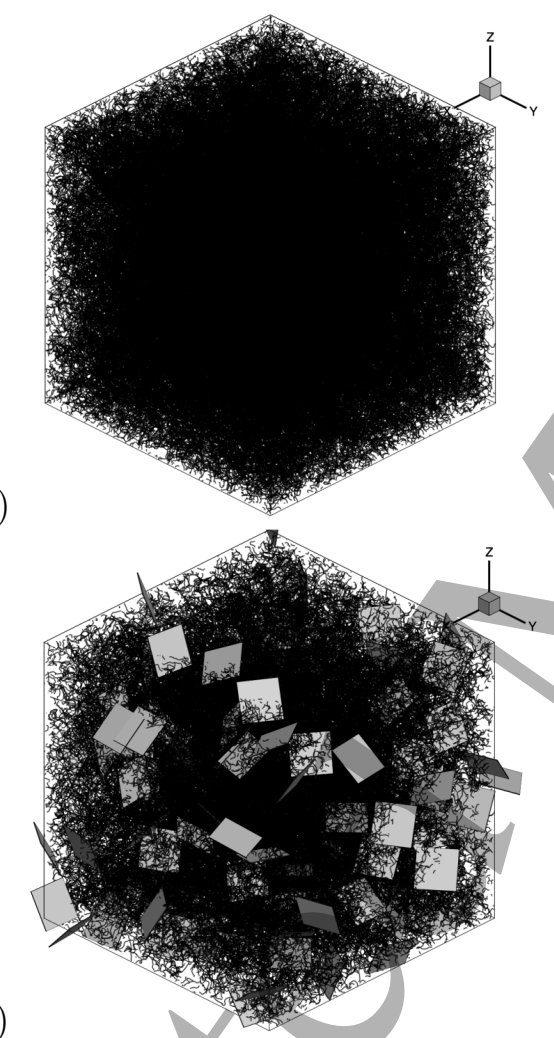

b)
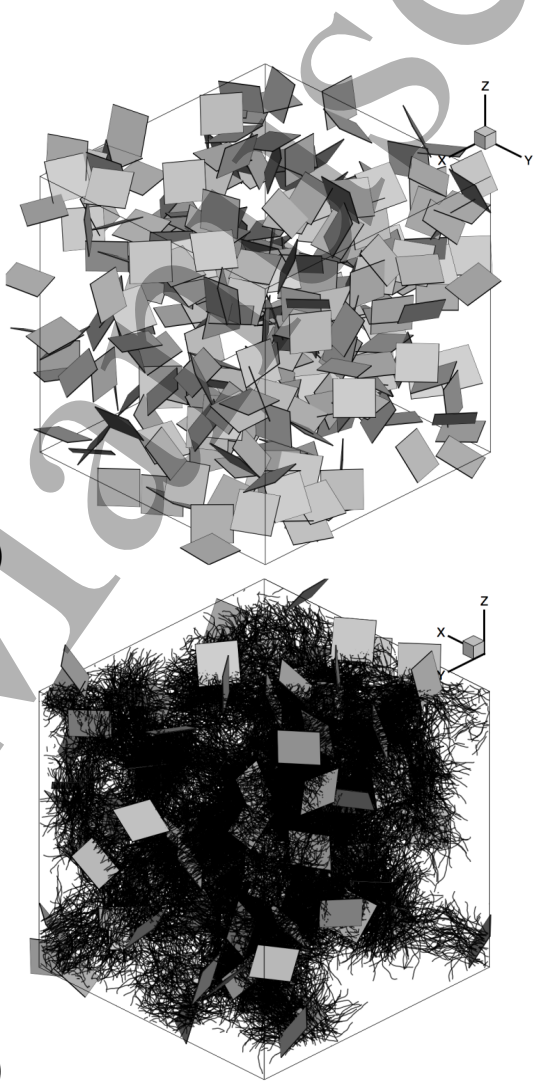

Figure 8. Networks formed using (a) CNTs, (b) GNPs, (c) mixed CNTs and GNPs, and (d) hybrid particles. In all four cases, the nanofiller content is $0.75 \%$ volume fraction.

From the foun types of nanofillers, only the hybrid particles formed a percolated network at the $0.25 \%$ volume fraction. In Figure $7 \mathrm{a}$, we present the backbone of the network, formed by the CNT bundles, from Figure 6d, while Figure 7b shows a second orientation of Figure 7a. We observe that the hybrid-particle network and its backbone 
a)

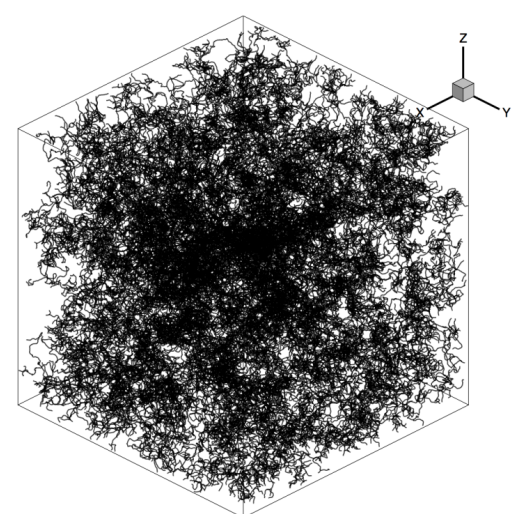

b)

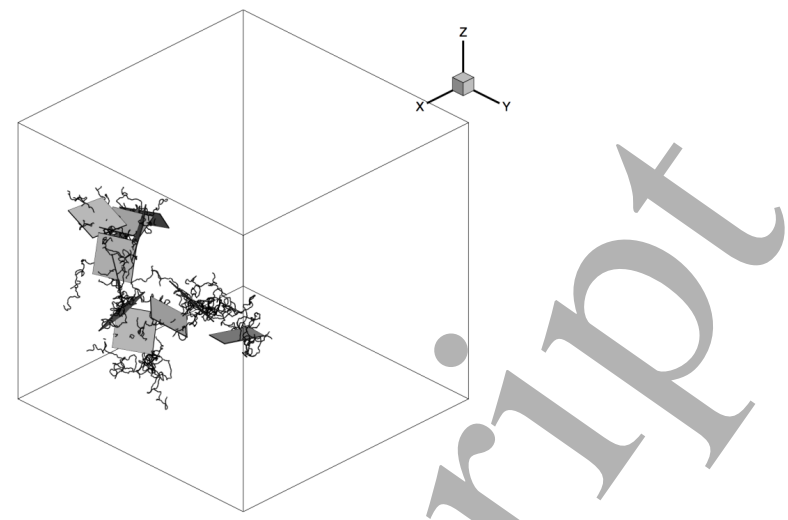

c)

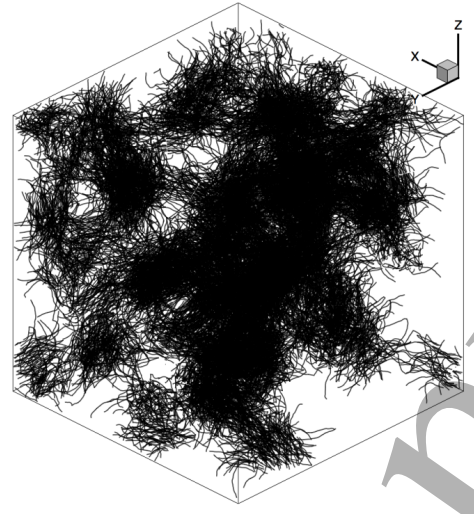

Figure 9. Backbones of the networks formed by (a) CNTs (corresponding to Figure 8a), (b) mixed CNTs and GNPs (Figure 8b), and (c) hybrid particles (Figure 8d). The network formed by GNPs did not percolate.

have the geometry of a segregated structure (e.g,, Figure 2 in [17]).

In Figure 8, we present networks for the four types of nanofillers using $0.75 \%$ volume fraction. At this volume fraction, only the GNP network does not percolate. As in Figure 8, the samples with only CNTs, GNPs, and a mix of the two show homogeneous distributions of the nanofillers (Figures 8a, 8b, and 8c, respectively). In Figure 8d, the distribution of the hybrid particles also appears homogeneous, in contrast to Figure $6 \mathrm{~d}$. This homogeneity comes from the greater number of particles inside the sample.

The backbones of the networks from Figure 8 are presented in Figure 9; as the GNP sample does not form a percolated network, it is not shown. The backbone formed by only CNTs has a homogeneous distribution (Figure 9a). The backbone formed by a mix of CNTs and GNPs appears to form a conductive path in Figure 9b, similar to those in Figure 7. However, since the CNTs in the mixed sample are not concentrated on the GNP surfaces as in a segregated structure, the CNTs are less likely to have contact 


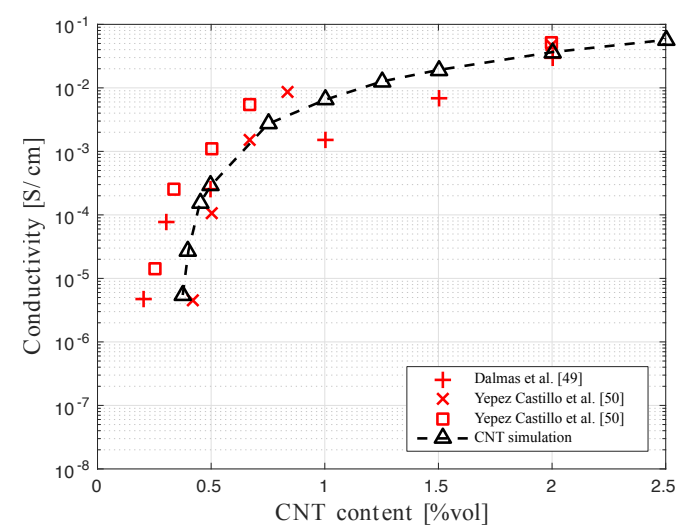

Figure 10. Comparison electrical conductivity results from our simulations and those reported in the literature.

with each other. Thus, this conductive path is formed by fewer CNTs than the paths formed by hybrid particles. Finally, we present the backbone of the network formed by hybrid particles in Figure 9c, where the GNPs are omitted from the figure to better visualize the structure of the network. We observe that the conductive paths are still formed by the CNT bundles on the GNPs. These bundles of CNTs are formed thanks to the GNPs concentrating CNTs and increasing locally the CNT volume content. Thus, the backbone of the hybrid-particle network at $0.75 \%$ volume fraction also has a segregated structure.

\subsection{Comparison with experiments}

We compare the electrical conductivity results obtained from our model with those reported in the literature (Figure 10). Since experimental data for polymers loaded with hybrid particles are scarce, we perform the validation using only our electrical conductivity results from the CNT-loaded polymers. The difference in electrical conductivity of the composites results from the nanofiller geometries. The numerical model and conductivity mechanisms are the same for both CNTs and hybrid particles. Therefore, we believe the numerical model presented here is applicable to composites filled with hybrid particles.

We run simulations using the same CNT geometries as those used for the CNTs grown on hybrid particles, i.e. $l_{C N T}=2-4 \mu \mathrm{m}$ and $r_{C N T}=7.6 \mathrm{~nm}$ following the typical geometry of CNTs in hybrid particles [24,31]. In this way, the results of the 


\begin{tabular}{lc} 
Table 1. & Percolation \\
threshold, $\phi_{c}$, for the four \\
studied nanofillers. \\
\hline Nanofiller & $\phi_{c}[\%]$ \\
\hline Hybrids & 0.24475 \\
CNTs & 0.379 \\
Mixed & 0.38875 \\
GNPs & 1.3405 \\
\hline
\end{tabular}

samples with only CNTs are comparable to the samples with hybrid particles. We use volume fractions ranging from 0.35 to $2.5 \%$. We find that the electrical conductivity of the CNTs ranges from 0.2 to $10^{5} \mathrm{~S} / \mathrm{cm}[1,2]$. In our simulations, we use a middle-range conductivity (in a logarithmic scale) of $10^{2} \mathrm{~S} / \mathrm{cm}$. As a first approximation, we do not include the effects of curvature in the CNT's resistivity as it falls outside the scope of our paper. In addition, the curvature of CNTs is limited by the angle $\psi_{s}$. The values that this angle can take limit the CNT's curvature in such a way that the CNTs do not experience extreme bending. However, this could be an interesting research topic for our future work. De Vivo et al. [39] reported the junction resistance as a function of the thickness of the polymer between CNTs for different polymer work functions. Following that report, we assume a junction resistance of $10^{7} \Omega$, which corresponds to a polymer thickness and work function in the middle of the given ranges. Results from our simulations are represented with black triangles connected by a dashed line in Figure 10. Our simulation results reproduce the general trend observed in experimental data from the literature $[49,50]$, which are shown as unconnected red symbols in Figure 10. The CNTs used in the referenced experiments and the CNTs grown in hybrid particles are multi-walled CNTs. Also, the aspect ratios of those referenced CNTs are close to the ones used in our simulations. Therefore, we conclude that they are relevant experimental data to compare with our model.

\subsection{Improved electrical conductivity of the hybrid-particle network}

We run simulations of composites loaded with hybrid particles, CNTs, GNPs, and a mix of CNTs and GNPs to compare their efficiency and electrical conductivity. The geometries used for the CNTs and GNPs are, again, typical of hybrid particles [24,31]: $l_{C N T} \mp 2-4 \mu \mathrm{m}, r_{C N T}=7.6 \mathrm{~nm}, l_{G N P}=1-4 \mu \mathrm{m}$ and $t_{G N P}=30 \mathrm{~nm}$. Here, $l_{C N T}$ 

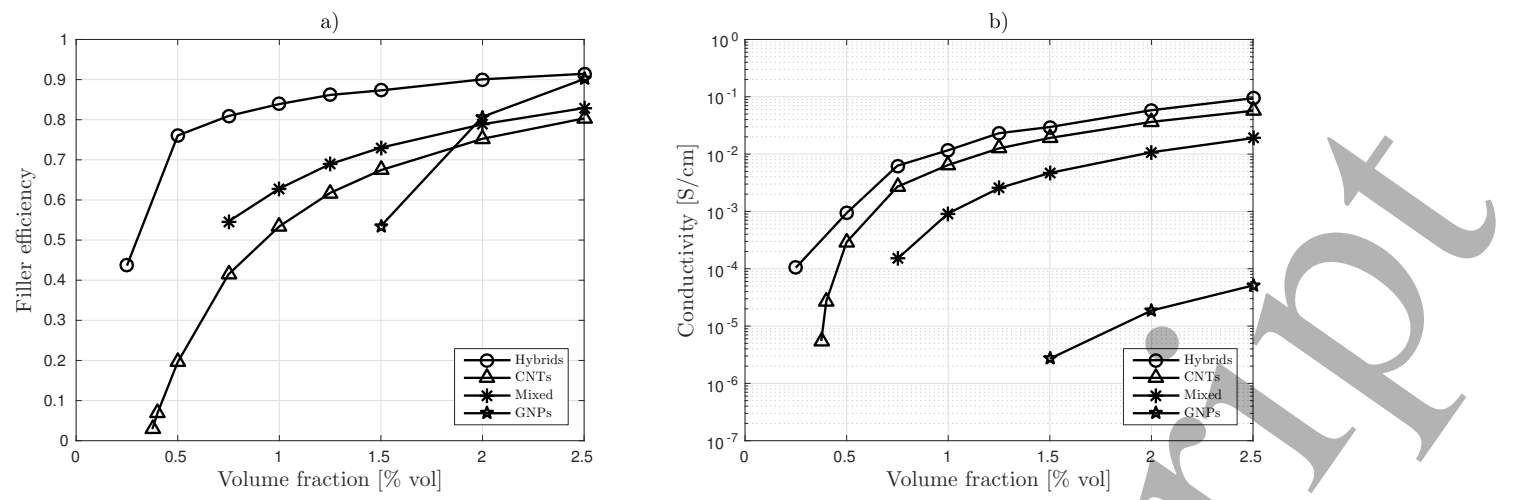

Figure 11. (a) Filler efficiencies at different volume contents. (b) Electrical conductivities for different fillers at different volume contents.

and $l_{G N P}$ follow a uniform distribution within their respective ranges. Regarding the electrical properties of the GNPs, their electrical conductivity parallel to the surface is in the range $6 \times 10^{2}-10^{5} \mathrm{~S} / \mathrm{cm}$, and around $1 \mathrm{~S} / \mathrm{cm}$ perpendicular to the thickness $[28,51,52]$. In our simulations, we used a middle-range conductivity (in a logarithmic scale) of $10^{3} \mathrm{~S} / \mathrm{cm}$ along the GNP surface and $1 \mathrm{~S} / \mathrm{cm}$ for the through-the-thickness conductivity.

In Table 1, we show the percolation thresholds that we obtained for all four types of nanofillers. The GNP network has the highest percolation threshold, whereas the hybrid-particle network has the lowest. GNP/polymer composites were reported to have a very high percolation threshold compáred to that of $\mathrm{CNT} /$ polymer composites $[3,4]$, just as our model predicts. It has been reported that straight and long CNTs reduce the percolation threshold of CNT networks [2,34]. In our observations of the individual hybrid particles, we find that the CNTs concentrated on GNP surfaces are less likely to curl. We consider this to be one of the reasons for the lower percolation threshold in hybrids. In addition, the CNTs grown on both surfaces of the GNPs can be considered bundles of long CNTs. Thus, the hybrid particle effectively behaves as a bundle of long CNTs, also contributing to the lower percolation threshold of the hybrid-particle network. We note that the main role of the GNPs in this network is to promote segregation, as they are the reason the CNTs are able to form and maintain bundles, reducing percolation threshold as described above.

The efficiencies (defined in section 2.2) of the four types of nanofillers at different 
volume fractions are presented in Figure 11a. We observe that the efficiency increases for all four types of fillers with the volume content. We also observe that the hybridparticle network has the highest efficiency for all volume fractions. However, the advantage of using hybrid particles is much higher at very low volume fractions. At higher volume fractions, most hybrid particles belong anyway to percolated networks and the efficiency is similar for all nanofillers. We attribute this higher efficiency to the bundles of CNTs: more CNTs are in contact with each other, increasing the overall efficiency. This means that GNPs increase the conductivity of the hybrid-particle network by promoting segregation.

We now compare the electrical conductivities of the fillers (Figure 11b). We note that GNPs show a low electrical conductivity compared with their efficiency. The samples with hybrid particles show the highest electrical conductivity for all volume contents. This reinforces that the segregation caused by the hybrid-particle networks improves their conductivity by bundling the CNTs and making the network more efficient, which makes it more conductive. In addition, we observe that the differences of electrical conductivity for the different fillers are similar to those observed in experiments. Because of this, and together with the observations of Section 3.2, we consider our model to be phenomenologically pertinent as it gives a plausible explanation for experimental observations and can now raise new questions about the materials under study.

\subsection{Optimization of the hybrid particle geometry}

In this section, we seek to further improve the electrical conductivity of polymers filled with hybrid particles by optimizing their geometry. We limit the optimization variables to the CNT and GNP aspect ratios and the density of CNTs on hybrids, $N$, which we define as the number of CNTs per micron squared. We define our search space based on the typical geometries of hybrids, mentioned in section 2.1. The CNT aspect ratio, $A R_{C N T}$, is modified by keeping the radius constant at $r_{C N T}=5 \mathrm{~nm}$ and varying the length within $3 \leq l_{C N T} \leq 8 \mu \mathrm{m}$, resulting in $300 \leq A R_{C N T} \leq 800$. In the case of the GNPs, we maintain a constant side length of $l_{G N P}=3 \mu \mathrm{m}$, while we vary the thickness within $10 \leq t_{G N P} \leq 60 \mathrm{~nm}$. In this way, the GNP surface remains constant 
for CNT growth. We note that the GNP aspect ratio is typically defined as the larger dimension divided by the smaller dimension, i.e., $A R_{G N P}=l_{G N P} / t_{G N P}[3,53,54]$. However, this definition of the aspect ratio is more useful if the larger dimension is the one that varies. Here, since the smaller dimension, the thickness, is varied, we use the inverse of the aspect ratio, $I A R_{G N P}=1 / A R_{G N P}=t_{G N P} / l_{G N P}$, to describe the GNP shape, where a thin (thick) GNP corresponds to a small (large) $I A R_{G N P}$. Finally, we vary the CNT density in the range $10 \leq N \leq 100 \mathrm{CNTs} / \mu \mathrm{m}^{2}$. All simulations are performed using cubic samples of size $a=30 \mu \mathrm{m}$ and a carbon content of $1 \%$ volume fraction.

In Figure 12, we present a schematic of the changes in the microstructure of a polymer filled with hybrid particles as the particle geometry is modified. The initial microstructure is shown at the center of Figure 12 and the arrows indicate a transformation that consists in increasing $N, A R_{C N T}$ or $I A R_{G N P}$. Since the carbon content is kept constant, increasing any of the geometric parameters results in reducing the number of hybrid particles in the polymer (see Figure 12). Increasing $N$ results in hybrid particles with more CNTs on the GNP surfaces as indicated with transformation $T_{A}$ in Figure 12. Increasing $A R_{C N T}$ results in hybrid particles with longer CNTs as indicated with transformation $T_{B}$ in Figure 12. Finally, increasing $I A R_{G N P}$ results in hybrid particles with thicker GNPs as indicated with transformation $T_{C}$ in Figure 12.

In Figure 13a, we show a 3D plot of the electrical conductivity as a function of $A R_{C N T}$ and $N$, while $I A R_{G N P}$ is kept constant at 0.01 . In Figure $13 \mathrm{~b}$ we present the level curves of the 3D plot in Figure 13a. We included arrows in Figure 13b that indicate the changes in the microstructure (see Figure 12) happening when moving along the $x$ - and $y$-axis. From the 3D plot (Figure 13a), we observe that the electrical conductivity increases by increasing either the CNT density or $A R_{C N T}$. In polymers filled only with CNTs, increasing $A R_{C N T}$ reduces the percolation threshold, which results in higher conductivities $[2,34]$. Thus, it is expected the same happens with the CNTs in the hybrid particles. We also observe that increasing $N$ beyond $55 \mathrm{CNTs} / \mu \mathrm{m}^{2}$ does not result in a significant increase in electrical conductivity. The contour plot in Figure $13 \mathrm{~b}$ shows that, for a fixed CNT density, the optimal electrical conductivity is found by continuously increasing $A R_{C N T}$. However, the lower the CNT density, 


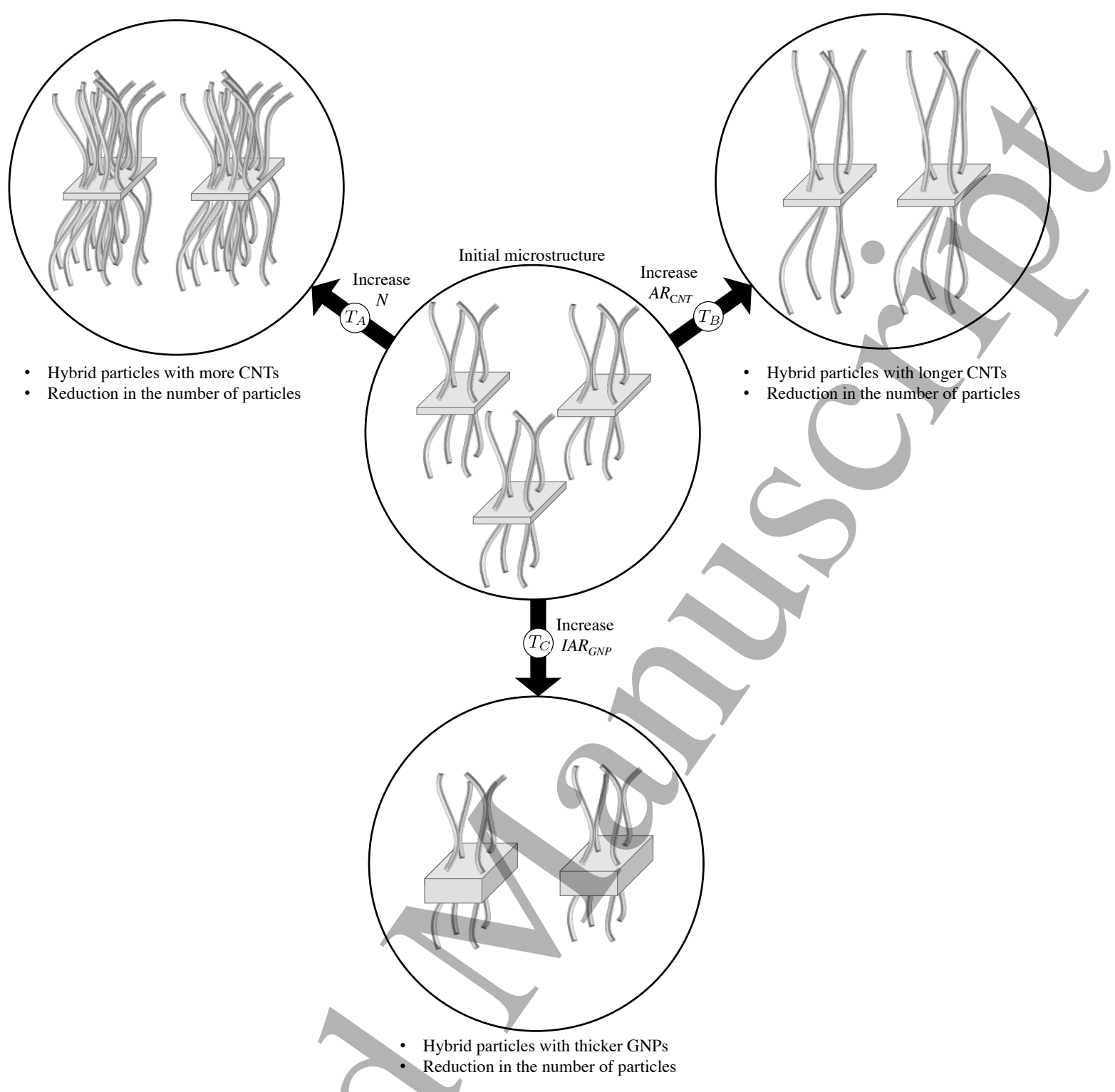

Figure 12. Schematic of the changes in the microstructure of a polymer filled with hybrid particles as the geometric parameters of the particles are modified. The initial microstructure is shown at the center and the arrows indicate a transformation $T_{A}\left(T_{B}\right.$ or $\left.T_{C}\right)$ of the microstructure that results from increasing $N\left(A R_{C N T}\right.$ or $\left.I A R_{G N P}\right)$. Since the carbon content is the same in both the initial and final microstructures, an increase in any of the geometric parameters results in the reduction of the number of hybrid particles in the polymer.

the smaller the change in electrical conductivity when increasing $A R_{C N T}$. Following the contour lines from the top left to the bottom right, we observe that the same conductivity can be achieved by having several short CNTs on GNP surfaces or a few long CNTs. These two routes can correspond to different design choices. Indeed, synthesizing longer CNTs usually results in larger synthesis time [23]. Long CNTs are also easier to break [55]. So we could expect that having short CNTs will help 
maintaining the integrity of the hybrid particles when they are mixed with the polymer. These points need to be validated experimentally, but this illustrates how our simple model can help in guiding the design of such materials.

In Figure 14a, we show a 3D plot of the electrical conductivity as a function of $A R_{C N T}$ and $I A R_{G N P}$, while $N$ is kept constant at $25 \mathrm{CNTs} / \mu \mathrm{m}^{2}$. In Figure $14 \mathrm{~b}$ we present the level curves of the 3D plot in Figure 14a, where the arrows indicate the changes in the microstructure (see Figure 12) when moving along the $x$ - and $y$-axis. We observe in the $3 \mathrm{D}$ plot that the highest electrical conductivity is found when $A R_{C N T}$ is largest and $I A R_{G N P}$ is smallest. The same is observed from the contour plot in Figure 14b. Thus, a higher electrical conductivity can be achieved by continuously increasing $A R_{C N T}$ and continuously decreasing $I A R_{G N P}$. In other words, we expect the highest electrical conductivity to be achieved when CNTs make up most of the carbon content of the hybrid-particle network. This can be achieved by choosing thin GNPs as substrates for CNT growth. Of course, there will be a minimum thickness that allows the GNPs to have the rigidity to maintain the bundle of CNTs on their surfaces. Further experiments and analysis to determine such minimum thickness falls beyond the scope of this paper.

In Figure 15a, we show a 3D plot of the electrical conductivity as a function of $I A R_{G N P}$ and $N$, while $A R_{C N T}$ is kept constant at 500 . In Figure $15 \mathrm{~b}$ we present the level curves of the 3D plot in Figure 15a, where the arrows indicate the changes in the microstructure (see Figure 12) when moving along the $x$ - and $y$-axis. From Figure 15a, we observe that the highest electrical conductivity is achieved by increasing the CNT density and decreasing $I A R_{G N P}$. We also observe that increasing $N$ beyond 55 $\mathrm{CNTs} / \mu \mathrm{m}^{2}$ does not result in a significant increase in electrical conductivity, which is consistent with the results shown in Figure 13. For a fixed CNT density, the smallest $I A R_{G N P}$ results in the highest electrical conductivity, consistent with the results in Figure 14 .

In summary, we make the following recommendations that we expect will contribute to further increase the electrical conductivity of hybrid-particle networks. First, it is recommended to use thin GNPs so that the hybrid particles are synthesized in such a way that most of the carbon content is assigned to CNTs. Synthesis time could be 

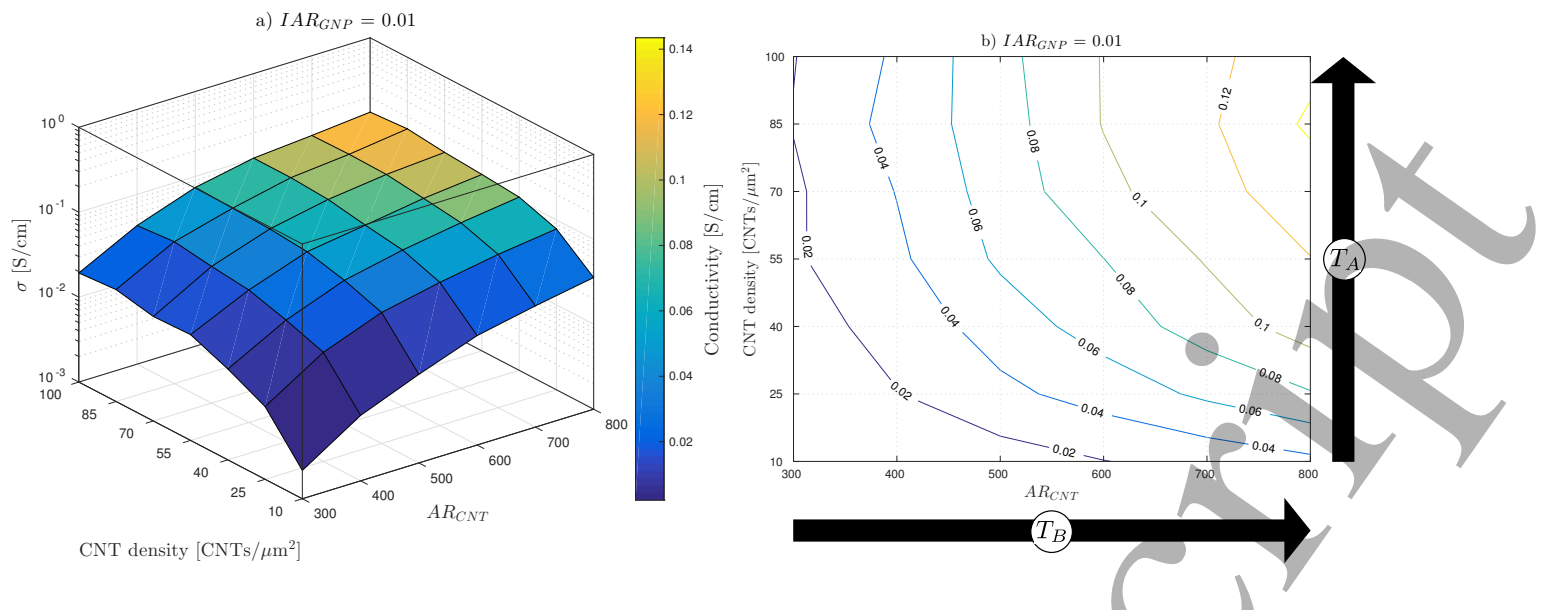

Figure 13. (a) $3 \mathrm{D}$ plot of electrical conductivity as a function of CNT aspect ratio, $A R_{C N T}$, and CNT density. GNP inverse aspect ratio, $I A R_{G N P}$, was kept constant at 0.01. (b) Contour plot of the 3D plot shown in (a), where transformation $T_{B}\left(T_{A}\right)$ applies when moving from left to right (bottom to top) along the $x$-axis $(y$-axis).

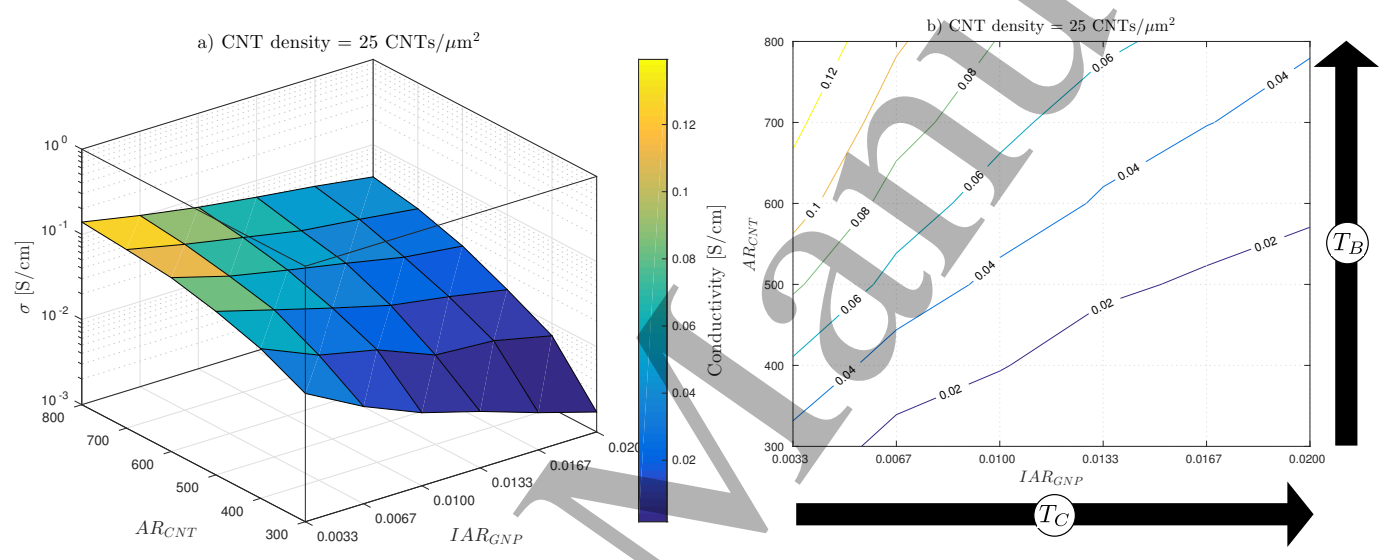

Figure 14. (a) $3 \mathrm{D}$ plot of electrical conductivity as a function of CNT aspect ratio, $A R_{C N T}$, and GNP inverse aspect ratio, IAR $R_{G N P}$. CNT density was kept constant at $25 \mathrm{CNTs} / \mu \mathrm{m}^{s}$. (b) Contour plot of the 3D plot shown in (a), where transformation $T_{C}\left(T_{B}\right)$ applies when moving from left to right (bottom to top) along the $x$-axis (y-axis).

reduced without sacrificing electrical properties of the composite by producing hybrid particles with a large CNT density of short CNTs. Also, from our results, we expect that increasing $N$ beyond $55 \mathrm{CNTs} / \mu \mathrm{m}^{2}$ will not result in a significant increase in electrical conductivity.

\section{Conclusions}

We developed a computational tool to reproduce geometrical representations of the recently developed hybrid-particle network obtained by growing CNTs on GNP sub- 

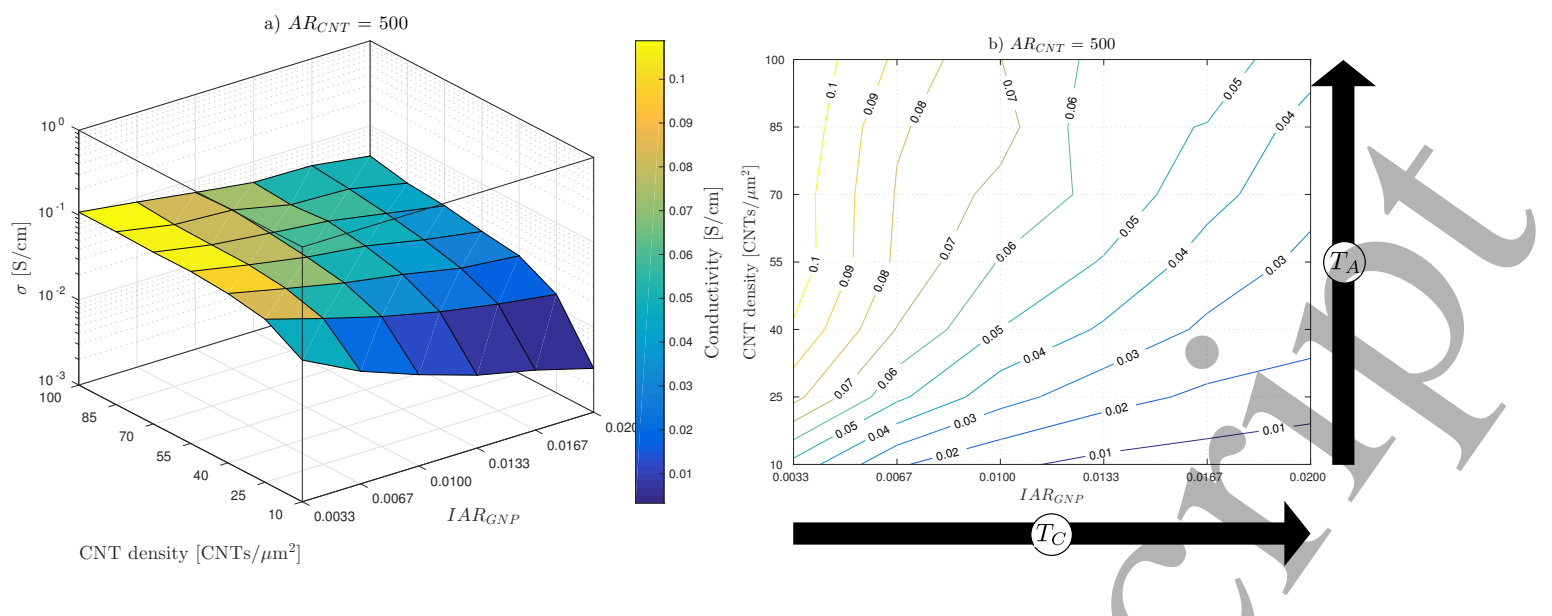

Figure 15. (a) $3 \mathrm{D}$ plot of electrical conductivity as a function of GNP inverse aspect ratio, $I A R_{G N P}$, and CNT density. CNT aspect ratio, $A R_{C N T}$, was kept constant at 500. (b) Contour plot of the 3D plot shown in (a), where transformation $T_{C}\left(T_{A}\right)$ applies when moving from left to right (bottom to top) along the $x$-axis (y-axis).

strates. We also used our tool to reproduce networks formed by only CNTs, only GNPs, and a mix of CNTs and GNPs.

By studying its morphology, we determined that the hybrid-particle network formed by bundles of CNTs concentrated on the GNPs is a segregated structure. Thus, the main role of the GNP is to promote segregation in the hybrid-particle network. The lower percolation threshold was caused by the CNTs being locally longer and straighter when grown on the GNPs. The increased electrical conductivity of the hybrid-particle network was explained by its higher efficiency compared to the other three studied networks. However, it was observed the advantage of using hybrid particles is higher at low volume fractions.

Finally, we investigated the geometry that optimizes the electrical conductivity of the hybrid-particle network. We recommend synthesizing hybrid particles in such a way that most of the carbon content is assigned to CNTs, as the main role of the CNTs is to contribute to the electrical conductivity whereas the main role of the GNPs is to ensure the creation of the segregated network. This study illustrates how relatively simple models can provide design inspiration for the improvement of such material. Further experimental investigation are of course needed to validate the role of each investigated parameter. 


\section{Funding}

The research reported in this publication was supported by funding from King Abdullah University of Science and Technology (KAUST).

\section{References}

[1] Wernik J, Meguid S. Recent Developments in Multifunctional Nanocomposites Using Carbon Nanotubes. Applied Mechanics Reviews. 2010;63(5):050801.

[2] Li C, Thostenson E, Chou TW. Sensors and actuators based on carbon nanotubes and their composites: A review. Composites Science and Technology. 2008;68(6):1227-1249.

[3] Li B, Zhong W. Review on polymer/graphite nanoplatelet nanocomposites. Journal of Materials Science. 2011;46(17):5595-5614.

[4] Sun X, Sun H, Li H, et al. Developing polymer composite materials: Carbon nanotubes or graphene? Advanced Materials. 2013;25(37):5153-5176.

[5] Mutiso R, Sherrott M, Rathmell A, et al. Integrating simúlations and experiments to predict sheet resistance and optical transmittance in nanowire films for transparent conductors. ACS nano. 2013;7(9):7654-63.

[6] Hashmi G, Miettunen K, Peltola T, et al. Review of materials and manufacturing options for large area flexible dye solar cells. Renewable and Sustainable Energy Reviews. 2011; 15(8):3717-3732.

[7] Hecht D, Hu L, Irvin/G. Emerging transparent electrodes based on thin films of carbon nanotubes, graphene, and metallic nanostructures. Advanced Materials. 2011; 23(13):1482-1513.

[8] Tai Y, Lubineau G. Double-Twisted Conductive Smart Threads Comprising a Homogeneously and a Grådient-Coated Thread for Multidimensional Flexible Pressure-Sensing Devices. Advanced Functional Materials. 2016;26(23):4078-4084.

[9] Oliva-Avilés A, Avilés F, Seidel G, et al. On the contribution of carbon nanotube deformation to piezoresistivity of carbon nanotube/polymer composites. Composites Part B: Engineering. 2013;47:200-206.

[10] Zhou J, Yu H, Xu X, et al. Ultrasensitive, Stretchable Strain Sensors Based on Fragmented Carbon Nanotube Papers. ACS Applied Materials and Interfaces. 2017;9(5):4835-4842.

[11] Aguilar Ventura I, Zhou J, Lubineau G. Drastic modification of the piezoresistive behavior of polymer nanocomposites by using conductive polymer coatings. Composites Science 
and Technology. 2015;117:342-350.

[12] Stauffer D, Bunde A. Introduction to Percolation Theory. Vol. 40. ; 1987.

[13] Li C, Thostenson E, Chou TW. Dominant role of tunneling resistance in the electrical conductivity of carbon nanotube-based composites. Applied Physics Letters. 2007; $91(22): 223114$.

[14] Sun X, Song M. Highly conductive carbon nanotube/polymer nanocomposites achievable? Macromolecular Theory and Simulations. 2009;18(3):155-161.

[15] Al-Saleh M, Sundararaj U. An innovative method to reduce percolation threshold of carbon black filled immiscible polymer blends. Composites Part A: Applied Science and Manufacturing. 2008;39(2):284-293.

[16] Grunlan J, Gerberich W, Francis L. Lowering the percolation threshold of conductive composites using particulate polymer microstructure. Journal of Applied Polymer Science. 2001;80(4):692-705.

[17] Pang H, Xu L, Yan D, et al. Conductive polymer composites with segregated structures. Progress in Polymer Science. 2014;39(11):1908-1933.

[18] $\mathrm{Hu} \mathrm{H}$, Zhang G, Xiao L, et al. Preparation and/electrical conductivity of graphene/ultrahigh molecular weight polyethylene composites with a segregated structure. Carbon. 2012;50(12):4596-4599.

[19] Gupta S, Ou R, Gerhardt R. Effect of the Fabrication Method on the Electrical Properties of Poly ( acrylonitrile-co-butadiene-co-styrene )/ Carbon Black Composites. Journal of Electronic Materials. 2006;35(2):224-229.

[20] Du J, Zhao L, Zeng Y, et al. Comparison of electrical properties between multi-walled carbon nanotube and graphene nanosheet/high density polyethylene composites with a segregated network structure. Carbon. 2011;49(4):1094-1100.

[21] Dichiara A, Yuan J, Yao S, et al. Chemical Vapor Deposition Synthesis of Carbon Nanotube-Graphene Nanosheet Hybrids and Their Application in Polymer Composites. Journal of Nanoscience and Nanotechnology. 2012;12(9):6935-6940.

[22] Yu L, Kang H, Lim YS, et al. Electrically conductive epoxy nanocomposites with expanded graphite/carbon nanotube hybrid fillers prepared by direct hybridization. Journal of Nanoscience and Nanotechnology. 2014;14(12):9139-9142.

[23] Zhao H. Preparation and characterization of carbon micro/nano hybrids and their functional composites [dissertation]. Université Paris-Saclay; 2015.

[24] Zhao H, Bai J. Highly sensitive piezo-resistive graphite nanoplatelet-carbon nanotube 
hybrids/polydimethylsilicone composites with improved conductive network construction. ACS Applied Materials and Interfaces. 2015;7(18):9652-9659.

[25] Lubineau G, Mora A, Han F, et al. A morphological investigation of conductive networks in polymers loaded with carbon nanotubes. Computational Materials Science. 2017; $130: 21-38$.

[26] Han F, Azdoud Y, Lubineau G. Computational modeling of elastic properties of carbon nanotube/polymer composites with interphase regions. Part I: Micro-structural charace terization and geometric modeling. Computational Materials Science. 2014;81:641-651.

[27] King J, Klimek D, Miskioglu I, et al. Mechanical properties of graphene nanoplatelet/epoxy composites. Journal of Applied Polymer Science. 2013;128(6):42174223.

[28] Jang B, Zhamu A. Processing of nanographene platelets (NGPs) and NGP nanocomposites: A review. Journal of Materials Science. 2008;43(15):5092-5101.

[29] Bandaru P. Electrical Properties and Applications of Carbon Nanotube Structures. Journal of Nanoscience and Nanotechnology. 2007;7(4):1239-1267.

[30] Li J, Ma P, Chow WS, et al. Correlations between Percolation Threshold, Dispersion State, and Aspect Ratio of Carbon Nanotubes. Advanced Functional Materials. 2007; $17(16): 3207-3215$.

[31] Li W, Dichiara A, Bai J. Carbon nanotube-graphene nanoplatelet hybrids as highperformance multifunctional reinforcements in epoxy composites. Composites Science and Technology. 2013;74:221-227.

[32] Hoshen J, Kopelman R.Percolation and cluster distribution. I. Cluster multiple labeling technique and critical concentration algorithm. Physical Review B. 1976;14(8):3438-3445.

[33] Babalievski F. Cluster counting: The Hoshen-Kopelman algorithm vs. spanning tree approaches. International Journal of Modern Physics C. 1998;9(1):18.

[34] Mutiso R, Winey K. Electrical properties of polymer nanocomposites containing rod-like nanofillers. Progress in Polymer Science. 2015;40:63-84.

[35] Li C, Chou TW. A direct electrifying algorithm for backbone identification. Journal of Physics A: Mathematical and Theoretical. 2007;40(49):14679-14686.

[36] Li C, Chou TW. Precise determination of backbone structure and conductivity of 3D percolation networks by the direct electrifying algorithm. International Journal of Modern Physics C. 2009;20(800):422-433.

[37] Bowyer A. Computing dirichlet tessellations. Computer Journal. 1981;24:162-166. 
[38] Watson D. Computing the n-dimensional delaunay tessellation with application to voronoi polytopes. The Computer Journal. 1981;24:167-172.

[39] De Vivo B, Lamberti P, Spinelli G, et al. Numerical investigation on the influence factors of the electrical properties of carbon nanotubes-filled composites. Journal of Applied Physics. 2013;113(24):244301.

[40] Deng F, Zheng QS. An analytical model of effective electrical conductivity of carbon nanotube composites. Applied Physics Letters. 2008;92(7):071902.

[41] Seidel GD, Lagoudas DC. A Micromechanics Model for the Electrical Conductivity of Nanotube-Polymer Nanocomposites. Journal of Composite Materials. 2009;43(9):917-941.

[42] Hu N, Karube Y, Yan C, et al. Tunneling effect in a polymer/carbon nanotube nanocomposite strain sensor. Acta Materialia. 2008;56(13):2929-2936.

[43] Li C, Chou TW. Electrical Conductivities of Composites with Aligned Carbon Nanotubes. Journal of Nanoscience and Nanotechnology. 2009;9(4):2518-2524.

[44] Buldum A, Lu J. Contact resistance between carbon nanotubes. Physical Review B. 2001; 63(16):161403-1-4.

[45] Fuhrer M. Crossed Nanotube Junctions. Science. 2000;288(5465):494-497.

[46] Li C, Thostenson E, Chou TW. Effect of nanotube waviness on the electrical conductivity of carbon nanotube-based composites. Composites Science and Technology. 2008; 68(6):1445-1452.

[47] Simoneau LP, Villeneuve J, Aguirre C, et al. Influence of statistical distributions on the electrical properties of disordered and aligned carbon nanotube networks. Journal of Applied Physics. 2013;114(11):114312.

[48] Yu Y, Song G, Sun L. Determinant role of tunneling resistance in electrical conductivity of polymer composites reinforced by well dispersed carbon nanotubes. Journal of Applied Physics. 2010;108(8):084319.

[49] Dalmas F, Cavaillé J, Gauthier C, et al. Viscoelastic behavior and electrical properties of flexible nanofiber filled polymer nanocomposites. Influence of processing conditions. Composites Science and Technology. 2007;67(5):829-839.

[50] Yepez Castillo F, Socher R, Krause B, et al. Electrical, mechanical, and glass transition behavior of polycarbonate-based nanocomposites with different multi-walled carbon nanotubes. Polymer. 2011;52(17):3835-3845.

[51] Geng Y, Wang S, Kim J. Preparation of graphite nanoplatelets and graphene sheets. Journal of Colloid and Interface Science. 2009;336(2):592-598. 
[52] Strem Chemicals, Inc [Internet]. Graphene nanoplatelets ; Accessed May 24, 2017. Available from: https://www.strem.com/resource/5/literature_sheets-nanomaterials.

[53] Safdari M, Al-Haik M. Electrical conductivity of synergistically hybridized nanocomposites based on graphite nanoplatelets and carbon nanotubes. Nanotechnology. 2012; 23(40):405202.

[54] Chen G, Weng W, Wu D, et al. PMMA/graphite nanosheets composite and its conducting properties. European Polymer Journal. 2003;39(12):2329-2335.

[55] Otten R, Van Der Schoot P. Connectivity percolation of polydisperse anisotropic nanofillers. Journal of Chemical Physics. 2011;134(9). 